\title{
Influence of Barrier Width on Spin-Polarisation Measured by Point Contact Andreev Reflection
}

\author{
P. Wójcik • A. Naylor • M. Wołoszyn • B.J. Hickey • \\ B.J. Spisak · J. Adamowski
}

Received: 7 September 2010 / Accepted: 8 September 2010 / Published online: 23 September 2010

(C) The Author(s) 2010. This article is published with open access at Springerlink.com

\begin{abstract}
A modification of the Blonder-Tinkham-Klapwijk (BTK) model is proposed for the description of the ferromagnet-superconductor interface. Modelling the contact potential with a rectangular barrier, we investigate the influence of the barrier width at the interface on the value of spin polarisation measured by point contact Andreev reflection. Results suggesting that neglecting the width of the barrier at the interface can lead to an overestimation of the spin polarisation using the original BTK model are presented. This effect is particularly strong for low values of polarization and vanishes for high polarisation. The impact on analysis of the experimental data is also discussed.
\end{abstract}

Keywords Point contact Andreev reflection · Spin polarisation $\cdot$ PCAR $\cdot$ BTK theory

\section{Introduction}

Point contact Andreev reflection (PCAR) is an experimental techniques used to measure the electronic spin-polarisation at the Fermi level [1,2]. Although the idea of this method is relatively simple with no restriction on the sample geometry, the interpretation of experimental data requires careful analysis $[3,4]$.

P. Wójcik $(\bowtie) \cdot$ M. Wołoszyn · B.J. Spisak · J. Adamowski

Faculty of Physics and Applied Computer Science, AGH

University of Science and Technology, Al. Mickiewicza 30,

30-059 Kraków, Poland

e-mail:pelekwojcik@gmail.com

B.J. Spisak

e-mail: spisak@novell.ftj.agh.edu.pl

P. Wójcik · A. Naylor · B.J. Hickey

School of Physics and Astronomy, University of Leeds, Leeds LS2 9JT, UK
The interpretation of PCAR experimental data is based on formula derived from the BTK model [5] or its extensions $[6,7]$, modified to include a spin polarisation of the metal [1]. Typically, all these models assume that the ferromagnetsuperconductor (FM/SC) interfaces are described by the Dirac's delta potential with a finite transparency that is related to the microscopic properties in a non-trivial way [3]. The importance of this assumption when the spin polarisation is extracted in different transport regimes was discussed in detail by Woods et al. [4]. Nonetheless, it should be noted that the Dirac's delta in the BTK model is used to preserve analytical simplicity, and it cannot be regarded as a consequence of various physical phenomena at the real interface, in the general case. In this report, we slightly modify the original BTK model, namely we use a rectangular potential barrier instead of the Dirac's delta and we investigate the effect of a finite width of potential barrier on the value of polarisation $P_{\mathrm{BTK}}$ extracted from the BTK model in the ballistic limit. Our modification of the BTK model results from the fact that real FM/SC interfaces are imperfect, i.e. chemical, electronic, and structural mismatch between both materials may lead to the conclusion that the rectangular potential barrier is a better approximation for these complex situations. The main consequence of the model is that neglecting the width of the barrier at the FM/SC interface could lead to misinterpretation of experimental data using the BTK model in its original form, and consequently an incorrect value for the spin-polarisation. We verify the assumption by numerical simulations and we compare the result of simulations with the experimental data obtained for $\mathrm{Cu} / \mathrm{Nb}$ contact.

\section{Model and Method of Calculations}

PCAR measurements are made by forming a point contact between FM and SC, and measuring the conductance. In our 
model, we assume a rectangular barrier exists at the interface of the FM/SC point contact. This assumption allows us to include the interference phenomena at the FM/SC interface that are not taken into account in the case of a Dirac's delta potential. The potential barrier of the simulated contact is shown in Fig. 1.

The height $Z$ and width $d$ of the potential barrier are free parameters of the model. In the limiting case, when $d \rightarrow 0$, our model of the contact corresponds to the BTK model.

For a small bias voltage, the conductance through the metal-superconductor contact can be evaluated by the formula [3]

$$
\begin{aligned}
G_{N}(V ; d, Z, T) \\
=\frac{e^{2}}{\hbar} \int_{-\infty}^{\infty} d E \frac{\partial f_{F-D}(E-e V, T)}{\partial V} \\
\quad \times\left[1+A_{N}(E ; d, Z)-B_{N}(E ; d, Z)\right],
\end{aligned}
$$

where $V$ is the bias voltage, $f_{F-D}(E-e V, T)$ is the quasiequilibrium Fermi-Dirac distribution, and $A_{N}(E ; d, Z)$ and $B_{N}(E ; d, Z)$ are the Andreev reflection and normal reflection probabilities, respectively.

The probabilities $A_{N}(E ; d, Z)$ and $B_{N}(E ; d, Z)$ can be determined by solving the Bogoliubov-de Gennes equations [8]

$$
\left[\begin{array}{cc}
H_{0}(x) & \Delta(x) \\
\Delta^{*}(x) & -H_{0}^{*}(x)
\end{array}\right]\left[\begin{array}{l}
u(x) \\
v(x)
\end{array}\right]=E\left[\begin{array}{l}
u(x) \\
v(x)
\end{array}\right],
$$

where $\Delta(x)$ is the local energy gap, $E$ is the quasi-particle energy measured from the Fermi level $\mu_{F}$ and $H_{0}(x)$ is the one-particle Hamiltonian in the form

$H_{0}(x)=-\frac{\hbar^{2}}{2 m} \frac{d^{2}}{d x^{2}}+U(x)-\mu_{F}$

with the potential energy $U(x)=Z \Theta(x) \Theta(d-x)$ where $\Theta(x)$ is the Heaviside step. The rest of symbols have the usual meaning.

The BTK model with the Dirac's delta potential or the rectangular barrier potential can be generalized to include

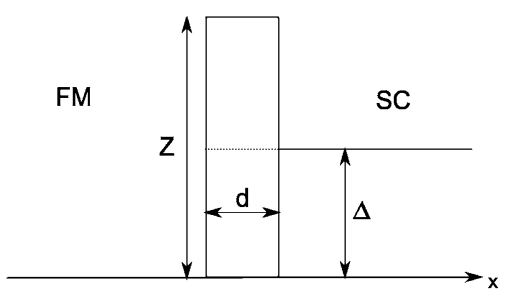

Fig. 1 Model potential used for the ferromagnet-superconductor (FM/SC) contact allowing to include quantum interference phenomena at the interface. Instead of Dirac's delta potential the rectangular potential is assumed. The height $Z$ and width $d$ of the potential barrier are free parameters of the model. $\Delta(x)$ is the local energy gap the spin-polarisation $P$. In this case, the total conductance of the contact is given by the formula

$$
\begin{aligned}
G(V ; d, Z, P)= & (1-P) G_{N}(V ; d, Z, P) \\
& +P G_{H}(V ; d, Z, P),
\end{aligned}
$$

where $(1-P) G_{N}(V ; d, Z, P)$ and $P G_{H}(V ; d, Z, P)$ are the fully unpolarised part and the fully polarised part of the total conductance, respectively. The fully polarised part is calculated by putting $A_{N}(E ; d, Z)=0$ in formula (1).

In practice, (4) is used to extract the values of spinpolarisation from the measurements of the total conductance by an appropriate fitting procedure $[6,7,9,10]$.

\section{Results}

Applying the scattering matrix method to solve the Bogoliubov-de Gennes equations, we found the probability amplitudes of Andreev and normal reflections as a function of the width of the potential barrier for different values of the parameter $Z$. In particular, Fig. 2 shows the oscillation of the Andreev reflection probability for a few values of parameter $Z$. These curves are obtained using $\Delta=1.5 \mathrm{meV}$ and $Z<\mu_{F}$, where $\mu_{F}=7 \mathrm{eV}$ corresponds to the Fermi energy in a typical metal. The amplitude and period of these oscillations depend on the value of the parameter $Z$, and moreover, the oscillations disappear as the quality of the FM/SC contact increases. On the other hand, for $Z>\mu_{F}$ we obtained an exponential decay of the Andreev reflection probability as a function of the potential barrier width.

As the normalised conductance of the point contact is determined by the probabilities for Andreev and normal reflection we therefore also expect an oscillation in conductance

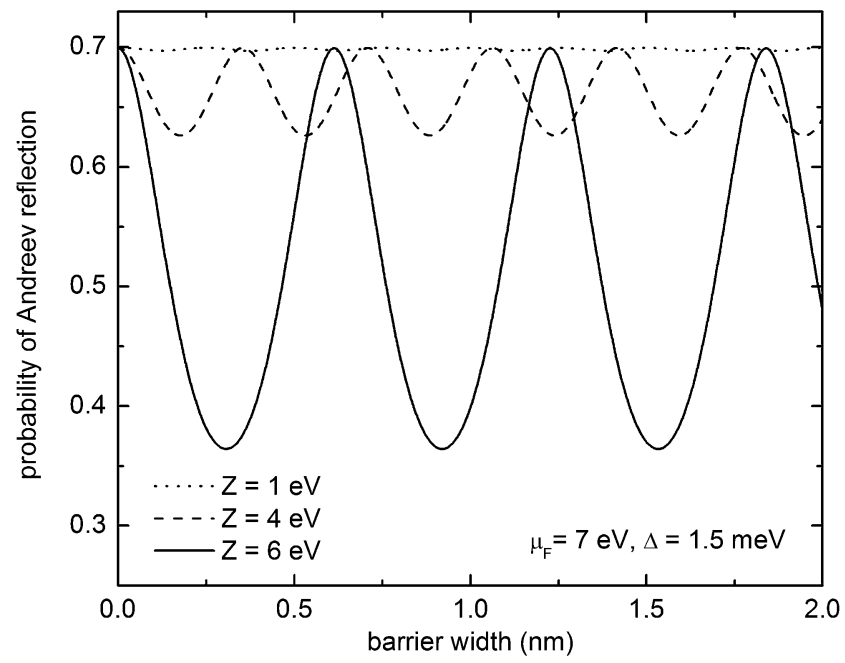

Fig. 2 Calculated Andreev reflection probability as a function of the width contact barrier for $Z<\mu_{F}$, where $\mu_{F}$ corresponds to the Fermi energy in a typical metal 

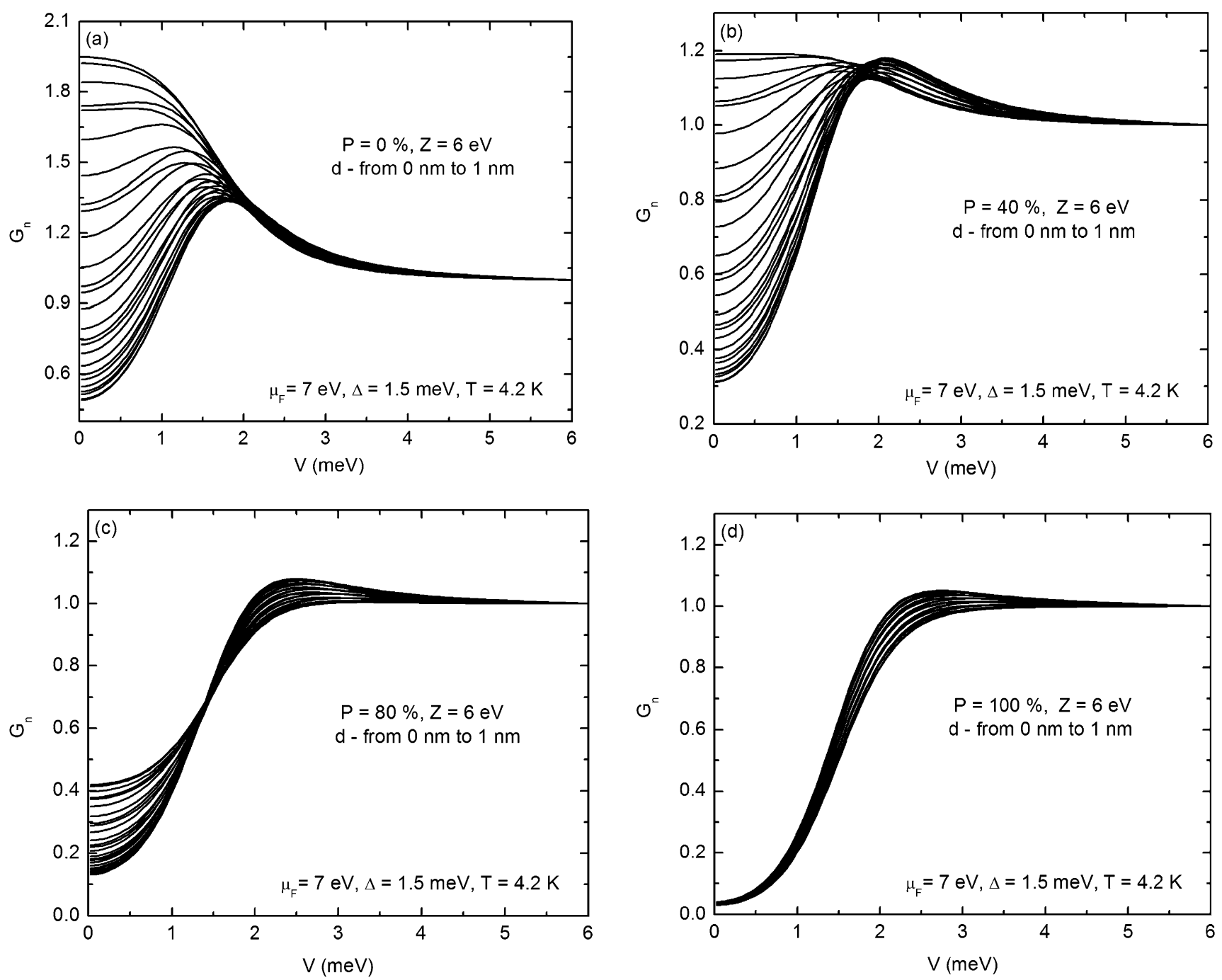

Fig. 3 Normalized conductance $G_{n}$ calculated for polarisation equal to (a) 0\%, (b) 40\%, (c) 80\%, and (d) $100 \%$. Results for the barrier width between 0 and $1 \mathrm{~nm}$ with step $0.04 \mathrm{~nm}$

as a function of barrier width and consequently a range of $G_{n}(V)$ curves for fixed spin-polarization $P$ and different barrier width. We performed numerical calculations of the total conductance as a function of the bias voltage for the widths of the barrier between 0 and $1 \mathrm{~nm}$ assuming four different values of the spin-polarisation, namely $0,40,80$, and $100 \%$. To discuss the situation when the oscillations are strongest, the parameter $Z$ was taken equal to $6 \mathrm{eV}$, see Fig. 2. The results of these calculations are shown in Fig. 3.

As a consequence of the periodic nature of the Andreev reflections presented in Fig. 2, we obtain a family of curves describing the conductance as a function of the applied bias voltage. All of the curves are placed between ones corresponding to the minimum and maximum values of the Andreev reflection probability. However, with increasing polarisation the influence of the barrier width becomes less significant. For example, if we compare the results for polarisation
$P=0$ (Fig. 3(a)) with $P=100 \%$ (Fig. 3(d)), we see a far greater variation in the shape of the conductance curve for the lower value of polarisation. Moreover, the curves presented in Fig. 3 cannot be obtained from the original BTK model with the same spin polarization and a varying value for $Z$.

In order to show this we have fitted the curves presented in Fig. 3 for $P=0$ and $80 \%$ and different barrier widths using the BTK model [7]. In the fitting procedure, we treat $P_{\mathrm{BTK}}, Z, \omega, \Delta$ as free fitting parameters, where $\Delta$ is the superconducting energy gap and $\omega$ is a broadening parameter [3]. It was found that the spin polarisation $P_{\mathrm{BTK}}$ from the BTK model can strongly depend on the barrier width, which is not controlled in the experiment, and could be completely different from spin polarisation $P$ for fitted theoretical curves calculated using the model with a rectangular potential barrier. For an unpolarised sample, the value for 

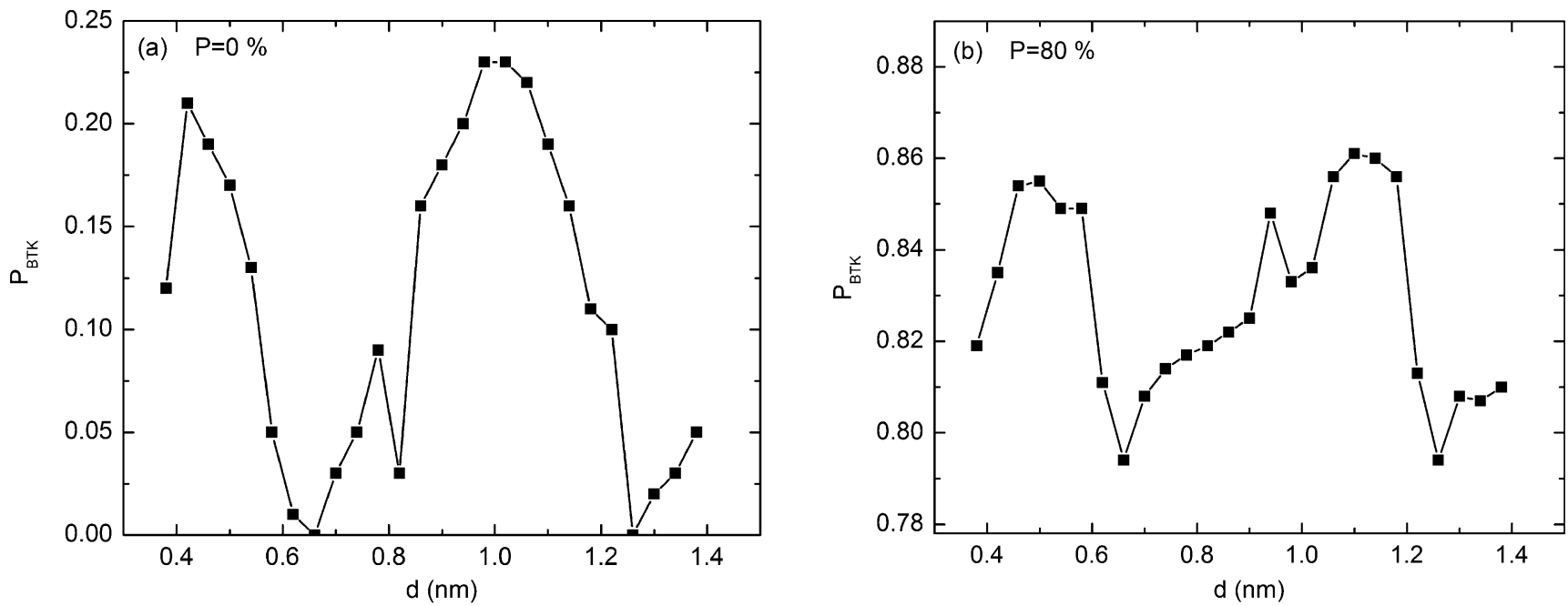

Fig. 4 Spin polarisation $P_{\mathrm{BTK}}$ from the BTK model obtained via fitting of theoretical curves as a function of the barrier width in case of polarization (a) $P=0$ and (b) $P=80 \%$. Theoretical curves are calculated using the model with rectangular barrier potential
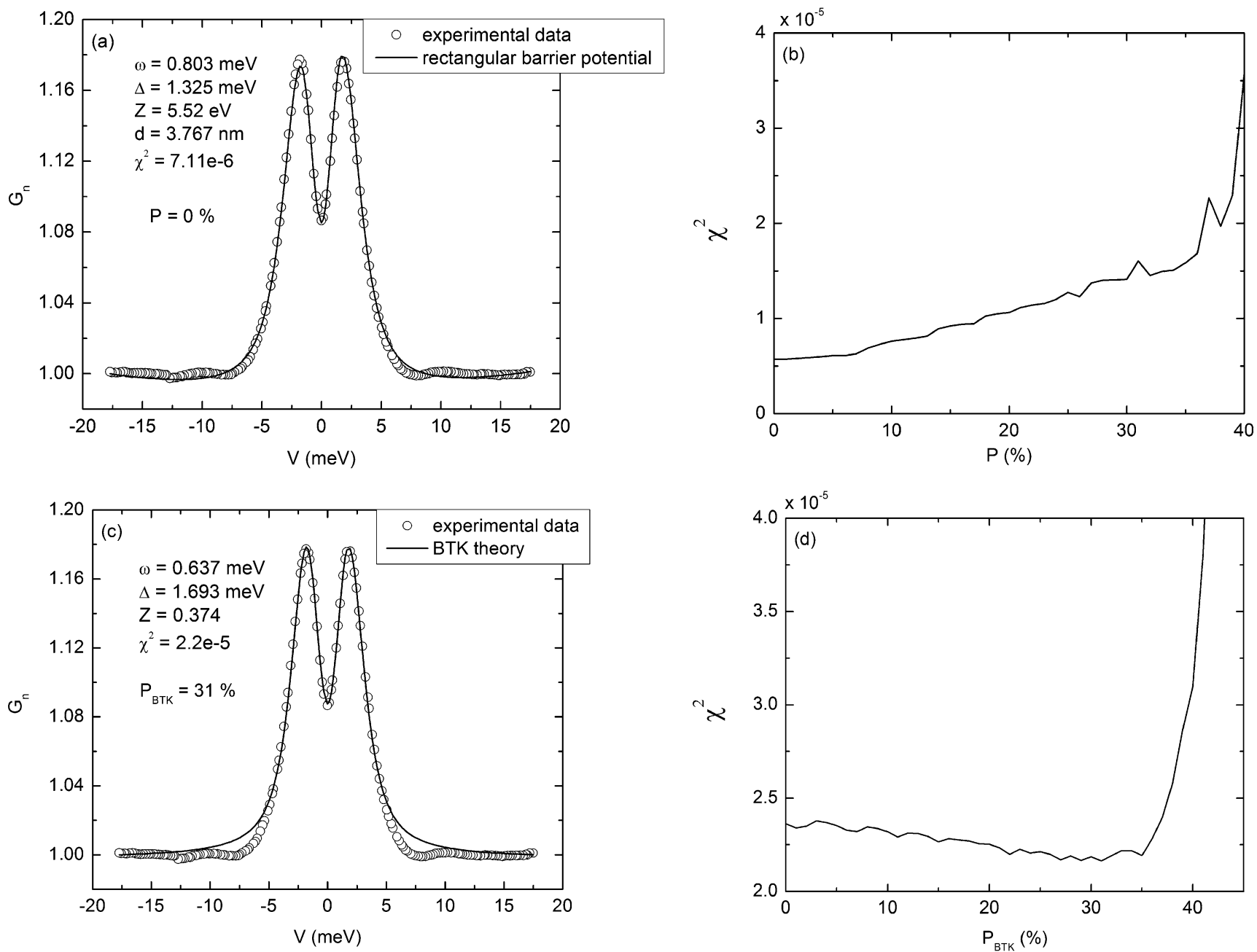

Fig. 5 Measured (dot line) and calculated (solid line) conductance together with the goodness-of-fit $\chi^{2}$ value for (a), (b) rectangular barrier, and (c), (d) BTK model with Dirac's delta 
$P_{\mathrm{BTK}}$ resulting from the BTK fit can vary between 0 and $25 \%$ as the barrier width changes (Fig. 4(a)). A similar situation, but on a smaller scale, is observed for $P=80 \%$, with results $P_{\mathrm{BTK}}$ between $80 \%$ and $85 \%$. Thus, we showed that neglecting the width of barrier at the FM/SC interface can lead to an overestimation of polarisation in analysis of experimental data when using the BTK model, and consequently to incorrect values of the spin-polarisation of ferromagnets. Moreover, the lower the spin-polarisation of the sample, the larger the error when using the BTK model to extract spin-polarisation. Since we are not able to control the width of the barrier in experiments, it can be responsible for ambiguous results, especially when measuring low spin-polarisation.

Direct evidence of the advantage arising from using the rectangular barrier is presented in Fig. 5 which shows a comparison between the calculated conductance and experimental data obtained for a $\mathrm{Cu} / \mathrm{Nb}$ junction. This particular case with polarization $P=0$ was chosen because according to our calculations the highest overestimation in analysis of experimental data using the BTK model occurs for low polarisation. The fitting procedure described in [3] was used to analyse the data. This involved fixing the value of spin polarization and letting the remaining parameters vary freely. This procedure was repeated for a range of values for the spin polarization-in this case between 0 and $40 \%$-and for each value the average deviation square between experimental data and fitting curves $\chi^{2}$ was calculated. A statistical 'best fit' is achieved if a clear minimum exists in the $\chi^{2}(P)$ function. In Fig. 5(b, d), $\chi^{2}$ as a function of spin polarisation for BTK model and the finite-width barrier case is presented. If the finite-width barrier is assumed (Fig. 5(a)), the fitting procedure gives $P=0$ as expected, in contrast to results from the original BTK model suggesting $P_{\mathrm{BTK}}=31 \%$ (Fig. 5(c)). Additionally, the accuracy of the fit is significantly better in the case of the rectangular barrier.

\section{Concluding Remarks}

In summary, we have investigated the influence of the potential barrier width on values of the spin-polarisation measured by point contact Andreev reflection.
Neglecting the width of the barrier could lead to an overestimation of the value of spin polarisation extracted from experimental data using the modified BTK model. The error is larger for lower values of spin polarisation. Including the barrier can yield appropriate values for the spin polarization, and in some cases, increase the statistical quality of the fitting procedure.

Acknowledgements This work was supported by the "Krakow Interdisciplinary Ph.D. Project in Nanoscience and Advanced Nanostructures" operated within the Foundation for Polish Science MPD Programme co-financed by the EU European Regional Development Fund and Physical Sciences Research Council (EPSRC) of the United Kingdom.

Open Access This article is distributed under the terms of the Creative Commons Attribution Noncommercial License which permits any noncommercial use, distribution, and reproduction in any medium, provided the original author(s) and source are credited.

\section{References}

1. Soulen, R.J., Byers, J.M., Osofsky, M.S., Nadgorny, B., Ambrose, T., Cheng, S.F., Broussard, P.R., Tanaka, C.T., Nowak, J., Moodera, J.S., Barry, A., Coey, J.M.D.: Science 282, 85 (1998)

2. Upadhyay, S.K., Palanisami, A., Louie, R.N., Buhrman, R.A.: Phys. Rev. Lett. 81, 15 (1998)

3. Boguslavsky, Y., Miyoshi, Y., Clowes, S.K., Branford, W.R., Lake, M., Brown, I., Caplin, A.D., Cohen, L.F.: Phys. Rev. B 71, 104523 (2005)

4. Woods, G.T., Soulen, R.J.Jr., Mazin, I., Nadgorny, B., Osofsky, M.S., Sanders, J., Srikanth, H., Egelhoff, W.F., Datla, R.: Phys. Rev. B 70, 054416 (2004)

5. Blonder, G.E., Tinkham, M., Klapwijk, T.M.: Phys. Rev. B 25, 4515 (1982)

6. Mazin, I.I., Golubov, A.A., Nadgorny, B.: J. Appl. Phys. 89, 11 (2001)

7. Strijkers, G.J., Ji, Y., Yang, F.Y., Chien, C.L.: Phys. Rev. B 63, $104510(2001)$

8. de Gennes, P.G.: Superconductivity of Metals and Alloys. Benjamin, New York (2001)

9. Ji, Y., Strijkers, G.J., Yang, F.Y., Chien, C.L., Byers, J.M., Anguelouch, A., Xiao, Gang, Gupta, A.: Phys. Rev. Lett. 86, 24 (2001)

10. Nadgorny, B., Mazin, I.I., Osofsky, M., Soulen, R.J.Jr., Broussard, P., Stroud, R.M., Singh, D.J., Harris, V.G., Arsenov, A., Mukovskii, Ya.: Phys. Rev. B 63, 184433 (2001) 